\title{
List of Contributing authors
}

\section{Debnarayan Jana}

Department of Physics

University of Calcutta

92 A.P.C Road

Kolkata -700009, India,

cujanad@yahoo.com

Chapter 1, 5

\section{Suman Chowdhury}

Department of Physics, Bangabasi College,

19, Rajkumar Chakraborty Sarani, Kolkata 700009, W.B., India, sumanchowdhury88@gmail.com

Chapter 1

\section{Lydia Rhyman}

University of Mauritius, Computational Chemistry Group, Department of Chemistry, Faculty of Science, 80837 Réduit, Mauritius, lyd.rhyman@gmail.com

Chapter 2, 8

\section{Mahir Tursun}

Department of Physics,

Science Faculty,

Anadolu University,

Eskişehir, 26470, Turkey

Chapter 2

\section{Hassan H. Abdallah}

Chemistry Department, College of Education,

Salahaddin University, Erbil, Iraq

Chapter 2

\section{Yee Siew Choong}

Institute for Research in Molecular Medicine (INFORMM),

Universiti Sains Malaysia,

Malaysia

Chapter 2

\section{Cemal Parlak}

Department of Physics, Science Faculty,

Ege University,

İzmir, Bornova, 35100,

Turkey

Chapter 2

\section{Prashant Kharkar}

SPP School of Pharmacy and Technology

Management,

SVKM's NMIMS,

V. L. Mehta Road, Vile Parle (West),

Mumbai-400056, India

Chapter 2

\section{Ponnadurai Ramasami}

University of Mauritius, Computational Chemistry Group, Department of Chemistry, Faculty of Science, 80837 Réduit, Mauritius p.ramasami@uom.ac.mu Chapter 2, 8, 11

\section{Selçuk Gümüş}

Faculty of Science, Department of Chemistry, Van Yuzuncu Yil University, 65080, Van, Turkey, gumuss@gmail.com Chapter 3

\section{Ayşegül Gümüş}

Faculty of Science, Department of Chemistry, Van Yuzuncu Yil University, 65080, Van, Turkey,

Chapter 3

\section{Winfred Mueni Mulwa}

Department of Physics, University of the Free State-Qwaqwa Campus, Private Bag x13, Phuthaditjhaba 9866, South Africa, mulwawinfred@gmail.com Chapter 4 


\section{Francis Birhanu Dejene}

Department of Physics,

University of the Free State-Qwaqwa Campus,

Private Bag x13,

Phuthaditjhaba 9866, South Africa,

Chapter 4

\section{Namrata Dhar}

Department of Physics

University of Calcutta

92 A.P.C Road

Kolkata -700009, India,

Chapter 5

\section{Rita Kakkar}

Computational Chemistry Laboratory,

Department of Chemistry,

University of Delhi,

Delhi 110007, India,

rkakkar@chemistry.du.ac.in,

Chapter 6

\section{Archa Gulati}

Computational Chemistry Laboratory,

Department of Chemistry,

University of Delhi,

Delhi 110007, India,

Chapter 6

\section{Cecil NM Ouma}

Faculty of Engineering, HySA Infrastructure $\mathrm{CoC}$, North-West University,

Private Bag X6001,

Potchefstroom, 2531, South Africa, moronaphtaly84@gmail.com

Chapter 7

\section{Walter E Meyer}

Department of Physics,

University of Pretoria,

Private Bag X20,

Pretoria 0028, South Africa

Chapter 7

\section{Helen Kavitha}

Department of Chemistry,

SRM University,

Ramapuram,

Chennai 600089,

Tamil Nadu, India,

helenkavithap@yahoo.co.in

Chapter 8

\section{Aleksey E. Kuznetsov}

Instituto de Química,

Universidade de São Paulo - SP,

Av. Prof. Lineu Prestes,

748 - Butantã,

CEP: 05508-000, São Paulo, SP, Brazil, aleksey73kuznets@gmail.com

Chapter 9

\section{Alcolea Palafox}

Departamento de Química-Física I, Facultad de Ciencias Químicas, Universidad Complutense,

Madrid, Spain,

alcolea@ucm.es

Chapter 10

\section{Siddheshwar D. Jadhav}

Department of Dyestuff Technology, Institute of Chemical Technology,

N. P. Marg, Matunga, Mumbai (MH)40001, India

Chapter 11

\section{Nagaiyan Sekar}

Department of Dyestuff Technology, Institute of Chemical Technology,

N. P. Marg, Matunga, Mumbai (MH)40001, India nethi.sekar@gmail.com

Chapter 11 